\title{
Charging our Electrical Devices in Anywhere and at Anytime
}

\author{
Carson Lam Kai Shun"
}

Faculty of Science Department of Mathematics, The University of Hong Kong, Pok Fu Lam, Hong Kong

DOI: $10.36347 /$ sipms.2020.v07i11.005

| Received: 14.11.2020 | Accepted: 26.11.2020 | Published: 30.11.2020

*Corresponding author: Carson Lam Kai Shun

Abstract

Original Research Article

Being able to charge electronic devices anywhere is a modern-day problem. That said, 'Made in China' external charging devices can solve this problem to some extent. However, these devices are often poor quality and can be a fire hazard. To address this issue, the author suggests using an electromagnetic spread spectrum, which is highly related to harvesting electrical energy from clean, renewable energy sources, and then transmitted to charge all types of electrical devices. By applying this author's proposed probability-random variables assignment - each type of electromagnetic wave is associated with a special channel with a centralised management information system controlled in the background. Such design can avoid EM-wave interference and encourage a delicate (or special) channel for energy transmitting. Hence, it can solve electrical devices charging problem or one may get the device charged anywhere and any time. Furthermore, with a professional electronic circuit design, together with metamaterials coated on the surface of a set antenna block, 90-100\% of harvested renewable-clean energy might be transmitted. There is an alternative way of charging by using inductive coils; however, there are some defects in such case. Further advance is the introduction of electromagnetic wormhole which can shield human beings from the influence of magnetic flux outside the tunnel during charging.

Keywords: electronic devices, Charging, electrical energy, EM-wave interference.

Copyright $\odot 2020$ The Author(s): This is an open-access article distributed under the terms of the Creative Commons Attribution 4.0 International License (CC BY-NC 4.0) which permits unrestricted use, distribution, and reproduction in any medium for non-commercial use provided the original author and source are credited.

\section{BACKGROUND}

In our modern world, energy has always been a problem of our human beings. This is because fossil fuel usually causes pollution. Clean energy sources such as wind and solar are still in the developing stages although they are expected to re- place fossil one. My proposal aims to study how one can convert those clean energy sources into electrical energy and then charging our usual electrical devices through common electromagnetic waves wirelessly such as either wi-fi, bluetooth or infra-red etc. My theoretical framework for the research is stated as follows:

\section{RESEARCH FRAMEWORK}

Consider the following imaginary statistical experiment that is often used when teaching statistics:
One should first toss three (or more) coins together in front of the participants, such that heads are on the front and tails are on back. The participants are then required to list all possible outcomes after tossing the coins.

In general, the set of all feasible results (outcomes) would be:

$\mathrm{S}=\{\mathrm{HHH}, \mathrm{HHT}, \mathrm{THH}, \mathrm{HTT}, \mathrm{THT}, \mathrm{TTH}$, TTT $\}$ (suppose one will obtain such expected result). There are also other combinations and permutations of heads and tails when each of them are assigned with a number. Hence, the outcome space is not just unique.)

Finally, the participants must count the number of tails for each possible outcome and then list them on a table, as seen below:

\begin{tabular}{|l|l|l|l|l|l|l|l|l|l|}
\hline Possible Outcomes $\left(\mathrm{s}_{\mathrm{i}}\right.$ ) & HHH & HHT & HTH & THH & HTT & HTT & TTH & TTT & \\
\hline No. of tails obtained $\mathrm{t}_{\mathrm{i}}$ & 0 & 1 & 1 & 1 & 2 & 2 & 2 & 3 & \\
\hline
\end{tabular}

(Obviously, the above table is NOT just the only one, there are lots of possible outcomes different ways of mapping tails. Instead of using tails, one may use no of heads for the mapping as an example.)
Consider a function $f$ (which should be called as the random variable). Intuitively, $f$ maps all the possible outcomes $\left(\mathrm{s}_{\mathrm{i}}\right)$ to the number of tails $\mathrm{T}_{\mathrm{i}}$ $f_{i}: \mathrm{s}_{\mathrm{i}} \longrightarrow \mathrm{t}_{\mathrm{i}}$ (i.e. no. of tails obtained as it takes 
values $\mathrm{t}=0,1,2,3 \ldots$ by $\mathrm{HKU}$, Dr. K.T. Leung's lecture note 1993-1994) or strictly speaking

$f: \mathrm{S} \longrightarrow \mathrm{T}$ (i.e. $\mathrm{R}-$ Real Number)

One may group the same random variables (with similar outcomes) to the home-used electromagnetic networks (such as wi-fi, RF, infra-red and Bluetooth etc.) respectively.

I.e. $0 \rightarrow$ Bluetooth; $1 \rightarrow \mathrm{Wi}-\mathrm{Fi} ; 2 \rightarrow$ infra-red; $3 \rightarrow$ RF (Radio Frequency);

Together with an Information management system (including a password login each network individually).

In our home environment (can also be extended for street / telephone booth), there are lots of electro-magnetic wave such as infra-red, wi-fi and RF together with Bluetooth etc. By introducing a management information system (MIS) (together with a password) to assign each EM wave with a suitable channel (as mentioned in the previous section), one can login into an individual network system once at a time without causing interference to each other. Upward a step, with the MIS and some meta-materials to the antenna, one can collect EM-wave (e.g. microwave). Although the received electronic signals may not be larger enough, if one can design a suitable rectifier circuit, it is feasible for us to employ the output D.C voltage to charge our electrical devices. That is one can convert the microwave energy backward to achieve our daily usage consumption.

The above plan is only feasible in theory. In practice, the induced current or voltage may be too low to charge any electrical device. In order to make the scheme possible, there should be a special / dedicated channel for the purpose of microwave to devices charging. Hence, the power output for charging devices and the power gained by these devices will be larger. Under my imaginary suggestion, there will be a combined way of collecting electricity from our nature resources like solar and wind etc. These energy are then stored in a charging batteries and will be used as a source for dedicated wifi charging. In addition, these stored energy are then used in places such as our common street light and telephone booth which used as the source of dedicated wi-fi for charging to our usual electrical devices with higher power of electricity output once logged in. Then both the low voltage and current problem will solved immediately once our government or telecommunication company is willing to investigate in a such unprecedented plan of proposed "microwave" charging project.

Therefore, the following is the expected procedure for charging an electrical device:

Step I: Clean Energy Source (Solar / wind energy etc) to charge batteries
Step II: Charged Batteries as sources of energy for special EM wave channel

Step III: Special EM wave channel sources in street light / telephone booth

Step IV: Login street light / telephone booth for wireless charge electrical devices Besides the charging procedure, there are also some practical questions concerning about the possibilities of my proposed charging scheme, thus here comes.

\section{RESEARCH QUESTIONS}

1. What kind of EM waves will give the maximum current and voltage output that is best suited for wireless charging?

2. How can we achieve the maximum gain of both current and voltage from the rectifier circuit for electrical devices charging? What are those factors influenced?

3. What is the energy convenience and efficiency for this kind of wireless charging scheme when compared with traditional fossil or only clean energy scheme? Does the proposed schedule feasible for us to implement?

\section{METHODOLOGY}

The main objectives or research aim of this study is to investigate the feasibility of wireless charging to electrical devices through a dedicated or special channel of EM (electro-magnetic) wave (come through street light / telephone booth) where the energy sources mainly from clean energy (such as solar / wind etc) to charge batteries. The core theory has been mentioned in the research framework section (tossing coin experiment) while the method of implementation can be employed through the EM wave meta material antenna (for receiving microwave only; other designs may be needed for different types of EM waves). The application domain is to use electronic circuit for the transportation of wireless induced current or voltage such that one is able to charge different kinds of electrical devices. The major methodologies that I used in this proposal are comparative, descriptive together with experimental one. It is because we want to compare people's past / present experience in the convenience and efficiency when using fossil fuels, unconverted clean energy and the suggested charging scheme. We also want to observe (as a means of collecting magnified current and voltage data) how well in descriptive way (what are those factors influence) will the electronic circuit provide a gain to both current and voltage. Finally, one will need to practically perform those experiments through isolating and controlling every relevant condition which determines the events investigated, so as to observe the effects when the conditions are manipulated. Then one can decide the feasibility of my proposed wireless charging scheme. 
Indeed, one may categorise the research paradigms (or models) into quantitative and qualitative approach. For the quantitative one, it is usually referred to the testing of theories, establishing facts together with the showing causal or other relationships between variables. This will be achieved via statistical, mathematical, or computational techniques. For the qualitative one, it is used for the developing theories, exploring a topic and developing a hypothesis. This can be realised through those scientific method of observation to obtain non-numerical data such as meanings, concepts of definitions characteristics, metaphors, symbols and description of things and not to their counts or measures [1]. Therefore, both of the comparative and descriptive methodologies are of qualitative types while the experimental one is quantitative kind. This research is then a mixed method of study.

When one is concerning about data collecting procedures or research design, one needs to use a questionnaire for the comparison of different experience in energy type of usage. Hence those non-essential characteristics of a phenomena can be eliminated by looking at multiple instance of it. It can be conducted at a marco (revolutions) or a micro level (individual experiences). Actually, the questionnaire is a set of questions.

Qualitative informations will be gathered from a particular population (most likely to be randomly selected electrical device users and student researcher as testers). Those responses will not be aggregated for analysis. The questionnaire items will have four response categories like "agree a lot", "agree a little", "disagree a little" and "disagree a lot" [2]. The major questions for normal users will focus in their experience of energy using - fossil, traditional clean energy and the present wireless charging where one will particularly study in their convenience of usage. While for those testers, the energy efficiency of the above three form of charging will also be questioned. I note that testers are those student researchers who will be answered in the energy efficiency questionnaire part's questions as the questionnaire is divided into two pats; one for ordinary user to question about convenience, the other is for student researchers (or testers) who will answer in the matters of energy efficiency. This is for the comparative research design.

For the descriptive research design, observations will be performed as a means of collecting qualitative data which attempt to examine those normal situations. One will also try to predict the outcome when the same circumstances happened. These observations will be written or recorded for the subsequently analysed. However, biased questions may result the distorted data in interviews, questionnaire and selective observation of events. In this study, observation is used to investigate what those factors that are influencing electric current is wirelessly transfered from past experienced mathematical data together with interviews to expert researchers. In fact, interviews offers a way for researchers to understand the relationships behind wireless current transportation and those influencing factors [2]. Furthermore, interviews are also well- known as a tool for understanding the reasons behind the gain and factors. I remark that Cool and Xie [4] used interviews as a supplementary way to survey and document choices. Hence, both relationships and reasons will be found between those investigated variables.

Lastly, there will be the practical experiment design with each factor being controlled individually to study the wireless transportation of received current from the electronic circuit. Causes and effects of their relationships will be the most interesting thing for me to research. Thus, correlation and ex post facto is implemented. Quantitative data will be collected in each of the cases. Actually, there are four types of experimental design. The first one is "Preexperimental" where one will made unreliable assumption without sufficient control over variables. The second one is "True experimental" where there will be a rigorous test in the similarity of groups before one is trying to test the influence of a variable to a sample of them when those circumstances are controlled. The third one is "Quasi- experimental" where only the shortcomings are identified without fulfilling all conditions of true experimental design. The last one is "Correlation and ex post facto" where correlation is used for the finding of cause and effect relationships between two sets of data; ex post facto is a reverse experimentation. It is employed to interprets the cause of phenomenon by observing its effects.

\section{Outcomes and Values}

The expected outcome of my research is that: it is feasible for us to make modifications to our street light and telephone booth for the wireless charging of electrical devices through a special EM wave channel from clean energy source's charged batteries. Although the energy efficiency may not be the best among fossil fuel and traditional clean energy, the proposed charging scheme is more convenient (where one can charge the electrical devices whenever he / she finds the street light / telephone booth) and safer (when compared with explosive external China made charging batteries). In addition, those common electronic symbols are employed to represent the rectifier circuit. Thus, it will easier for one to design, test and make amendments to it such that one can maximise the current and voltage obtained for wireless charging electrical devices whenever necessary. One of my design's advantage is that the device will only need an EM wave receiving antenna and rectifier circuit without buying and redesigning any new electrical devices. The most significant value of the scheme is one can charge any electrical devices anywhere in the world without 
carrying any other extra charging devices when the (modified or smart) street light or telephone booth exists for logging in with the known password.

\section{Limitations and Advantages over other charging}

There are also limitations to the above proposed charging scheme. First of all, the energy efficiency (about $37 \%$ of conversion, from Duke University, microwave experiment) may not be as good as expected. It is hard to convert all energy during the transmission between EM wave source and receiver since microwave signal propagates in all directions and the power will drop when the source sphere is extending larger (N.B. maximum power may be gained at the pole of it [5]. Obviously, there must be energy lost during transmission so there may be a need to redesign the antenna.

Furthermore, it may be hard to unify all standard of street light / telephone booth around our world's different countries. Hence, it is required to memorise various passwords which leads to the danger of lost. Finally, too strong EM wave power may cause harmful effect to our human life's healthy.

\section{A Comparison with charging through electric induction}

Theoretically, Maxwell equations tell us that if there is a time varying electric field, then a displacement current will be produced which is indeed proportional to the rate of change of the field. As a result, the induced current appears on the sender side will allow energy to be delivered among the space between different plates and hence act as role like a capacitor. When one is applying a load on the plates of the receiver side, there will be an induced electric charges moving between the side of the two plates. The result is mainly due to the time-varying displacement current moving around on the other side. This is known as "Capacitive Coupling". The aforementioned theory is widely used in our present daily mobile phone wireless charging. When one move up a stair, one may create an electromagnetic wormhole (which is a virtual one but not the physical space- time wormhole) with metamaterials and induced current on the plates of the other sides of the coupled capacitor. It is true that the technique can also avoid electromagnetic interference but there are several disadvantages over my proposed one. They are:

\section{Longer Charging Time}

If one provides the same power for the charging, most devices take $15 \%$ longer (in general) to charge. From the following graph, one may observe that wired charging is much faster (or the charging rate is better) than wireless charging for mobile phone. Thus, this author suggests that it is wise for us to charge electric devices directly. In my design, the energy source is a clean one, then it is transferred through electromagnetic waves (e.g. microwaves) to the receiver. The resultant electrical energy is charged directly to the electric devices. The whole process is faster.

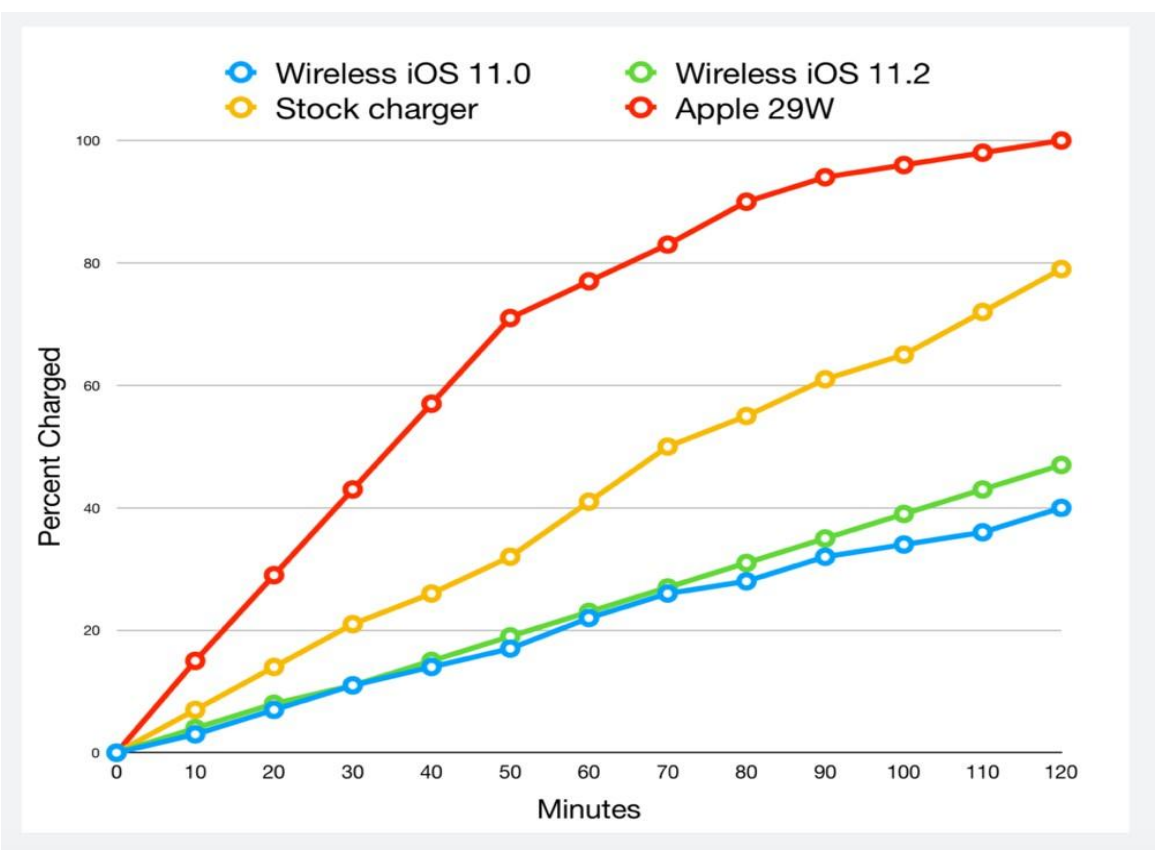

\section{More Money Is Spending}

It will increase the complexity of the charging process and manufacturing cost. This is because inductive charging needs drive electronics and coils in both sides of senders and receivers. This is practically known as resonance inductive charging. However, in my proposed design, it seems that only electronic circuits are required for receiving transmitted energy from the sender. The next part that connected to the 
devices is wired. It should be much simpler and cheaper.

\section{Poor Mobility}

In most cases, the present practical inductive pad's mobility is very poor. This is because people should place their phone statically on the induction pad without moving. However, for the wired charging, one may move the phone anywhere if the wire is long enough and even listen to the phone call. In my design, since the telephone booth and street light are all over any countries, it must be a convenient and safe method for charging outside homes.

\section{No Common Standard Exists}

Until recently, there is no common standard in the inductive charging standard. Different devices requires different charging devices. Devices may need to fulfil multiple standards. However, wired charging standard has been unified for some times which is not same as wireless one. My design employs wired charging which does not have the problem of standard.

\section{Low Efficiency}

The efficiency of wired charging is much higher than the wireless one. Moreover, devices may get hotter during inductive charging. This may harm the battery. In my design, there will be a warning signal when the device is full charged through wire. This can prevent it from over heated or charged.

\section{Miscellaneous for Receiver Electronic Circuits and Antenna Array Design:}

In order to achieve the maximum power harvesting, one should employ a set of Rectenna Array - Figure-3 [6] that coated by meta-material together with a matching circuit and resistance connections network rectifier - RCN (one of a similar case is shown in Figure $1 \& 2$ ) which should be designed by Advanced Design System (ADS) simulation software version 2017. The purpose of ADS is to design (by computation) a best and optimised power transfer circuit [7]. This is because in the rectifier circuits, either when there is a small change in the output load or if there is a change in the input power, this will produce changes in the matching of the rectifying device. Indeed these inputting matching changes will cause a degradation on the whole circuit performance [8]. That is all of the input energy in the port is transformed and transferred to the resistive load and hence can act ideally without the loss [9] whenever an $\mathrm{RCN}$ is introduced. I note that before the matching circuit is added, the maximum energy conversion efficiency is about $34 \%$ while when there is a matching, the conversion can be upgraded to $66.8 \%$ [7].

Next, to the matching or resistance connections network, there is a rectifier circuit that usually consists of Schottky diodes. It is well-known that the sensitivity of the circuit to convert the harvested
RF signals into a DC signal is directly related to the sensitivity of the used rectifying diode [10]. Hence, I suggest that one needs to further design one's own matching and a rectifier circuit for this project such that it will best fit those requirement (where one of a similar case study is shown in Figure-2).

Theoretically and under my imagination, by using a set of rectenna array together with the suitable meta-materials coated on their surface which connected with matching circuits and rectifier circuits, one may expect to receive nearly $90-100 \%$ of transmitted (microwave) energy. This author suggests the actual result should be experimented or obtained in the laboratory first by establishing a similar testing model before the practical implementation.

Finally, this author remarks that the proposed idea can be extended to a mini- electrical power station for charging electric transportations devices. Indeed, the station may replace our present oil stations in the future. This can reduce the air pollution in most of the highly commercial and densely populated cities like Hong Kong, New York and London etc as most of the oil cars will soon be diminished.

\section{Further Advances in Wireless Charging}

In the future, may we hope that there will be advances in the application of electromagnetic wormhole. It is actually not the physical wormhole that proposed in our cosmological theories for shortening the distance travelling in the universe. As this author may suggest in the research, the energy sender and the receiver antenna parts can establish an electromagnetic wormhole in between with suitable meta-material installed. Hence those magnetic sensitive apparatus from outside can be shielded in between them [11]. One more important thing is that we can manipulate how well the magnetic sensitive apparatus being exposed to the internal electromagnetic waves or even the permissibility of the man-made electromagnetic wormhole. This is because one may create the electromagnetic wormhole through controlling the amount of meta-material used externally on the surface of the shielded tunnel. Therefore, another application of the magnetic wormhole is: one may place a moving magnetic device (as the source) long distance away from the receiver side's conductor with the immediate part being magnetic wormhole shielded. An electric current will then be induced on the receiver side relative to an electrical load installed. Or electrical devices can be charged through magnetic resonance from long distance automatically. Thus, people are freeing from the claustrophobic environment of the magnetic resonance machine (or the source of resonance).

Conversely, one may imagine a thought experiment, instead of a transport vehicle's outside being shielded from magnetic flux, the vehicle's inside is shielded and people are seating in it. Then the 
magnetic resonance charging can be occurred outside the vehicle without cause any harmful effect to the human tissues. Hence, such vehicle can be charged wirelessly whenever and where-ever necessary. But the inside or the outside vehicle shielded experiments is actually a kind of philosophy with the practical possibilities.

\section{Appendix One - Electronic symbols and their} usage

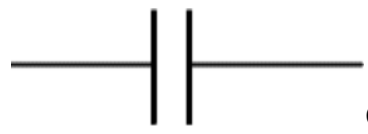

Capacitor: The normal use of capacitor is to store electric charge. It is worked with a resistor for a timing circuit. It also passes AC signals by blocking those DC signals. The role of it acts like a filter.

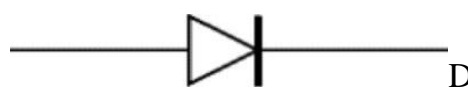

Diode: It is used in the circuit such that the current can only flow in a unique direction.
Resistor: It is used to resist the flowing of current through an electronic device and usually works with capacitor in a timing circuit.

$\psi$

Antenna: It is designed to receive and transmit radio signals. Meta- material may be painted on the surface of it for receiving and transforming EM wave into electric energy.

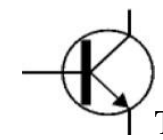

Transistor NPN: It is used to amplify current such that it works together with other components to become an amplifier or a switching circuit.

$\bigcap$ Inductor: It is a coil of wire that will induce a magnetic field when a current passes through it. It's another role is to convert electrical energy into mechanical one if one tries to pull something on it.

\section{Appendix Two - Referenced Rectifier Circuit for RF wave}

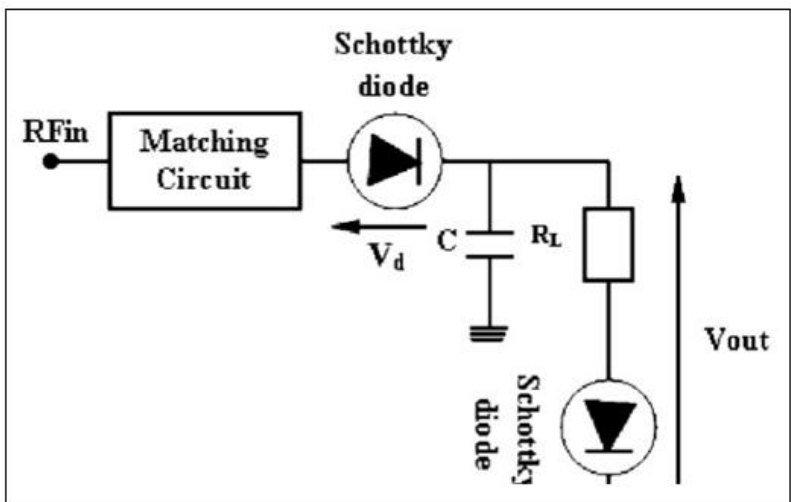

Figure on the left shows a series microwave rectifier detection sensitivity improvement configuration [12]

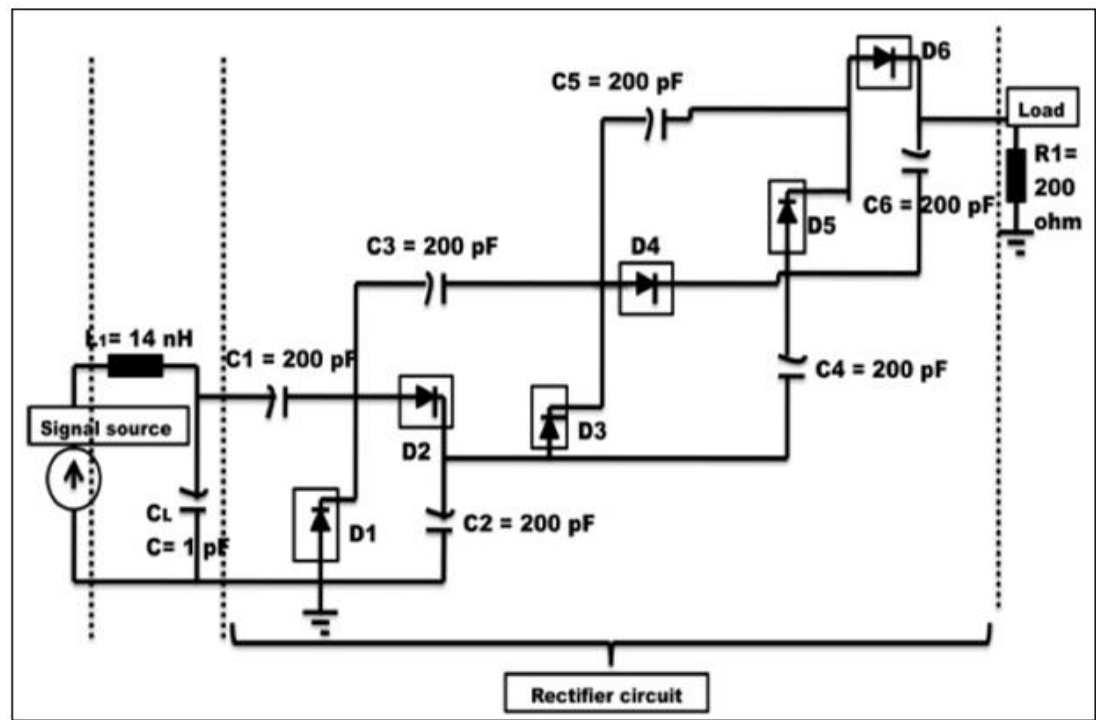

Figure on the left tells a three stage voltage doubler using Schottky diode HSMS 285B [5] 


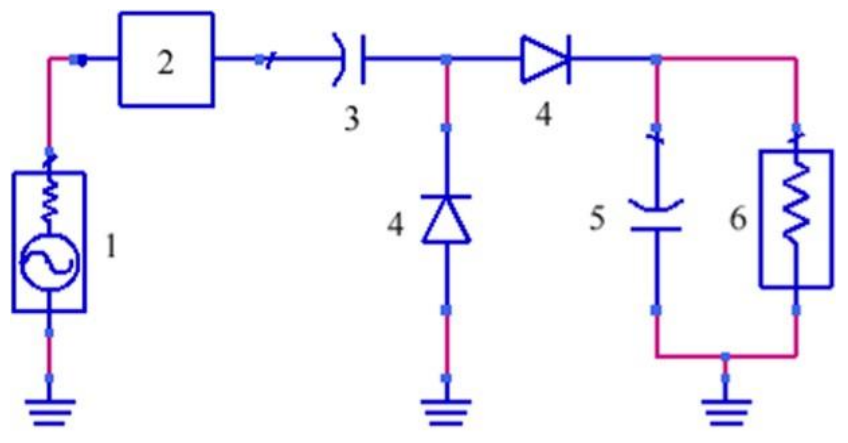
1. RF signal
2. SRR S-params
3. Matching cap
4. Greinacher doubler
5. Smoothing cap
6. Load

Figure on the left describes ADS simulation schematic of SRR power harvester. CST Microwave Studio was used to determine the SRR S- parameters [13].

12. Appendix Three - Proposed Clean Energy Charging Scheme for Street light / Telephone Booth

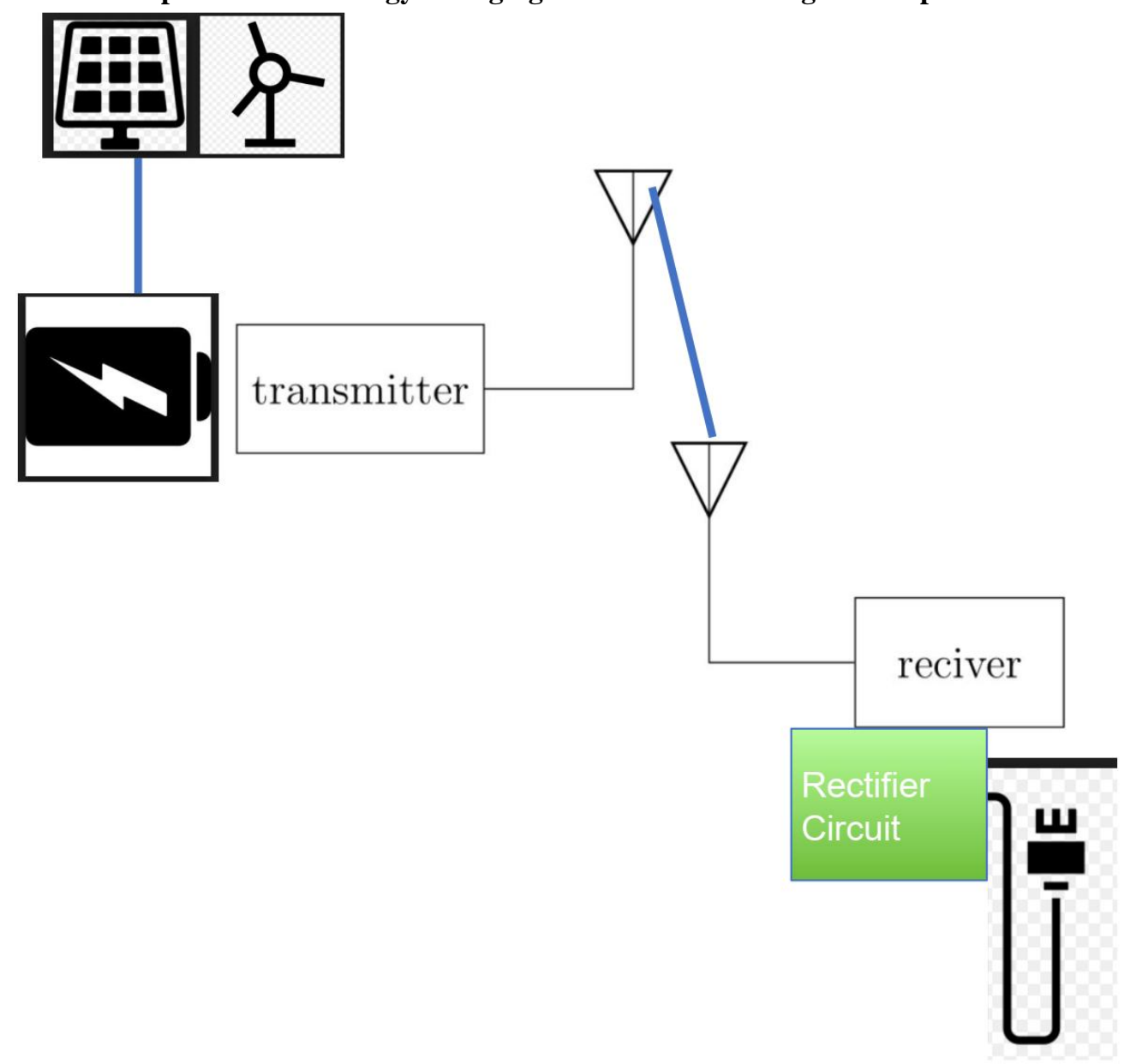

13. Appendix Four - A Suggested Information Management System for Logging into Special EM Wave Channel 

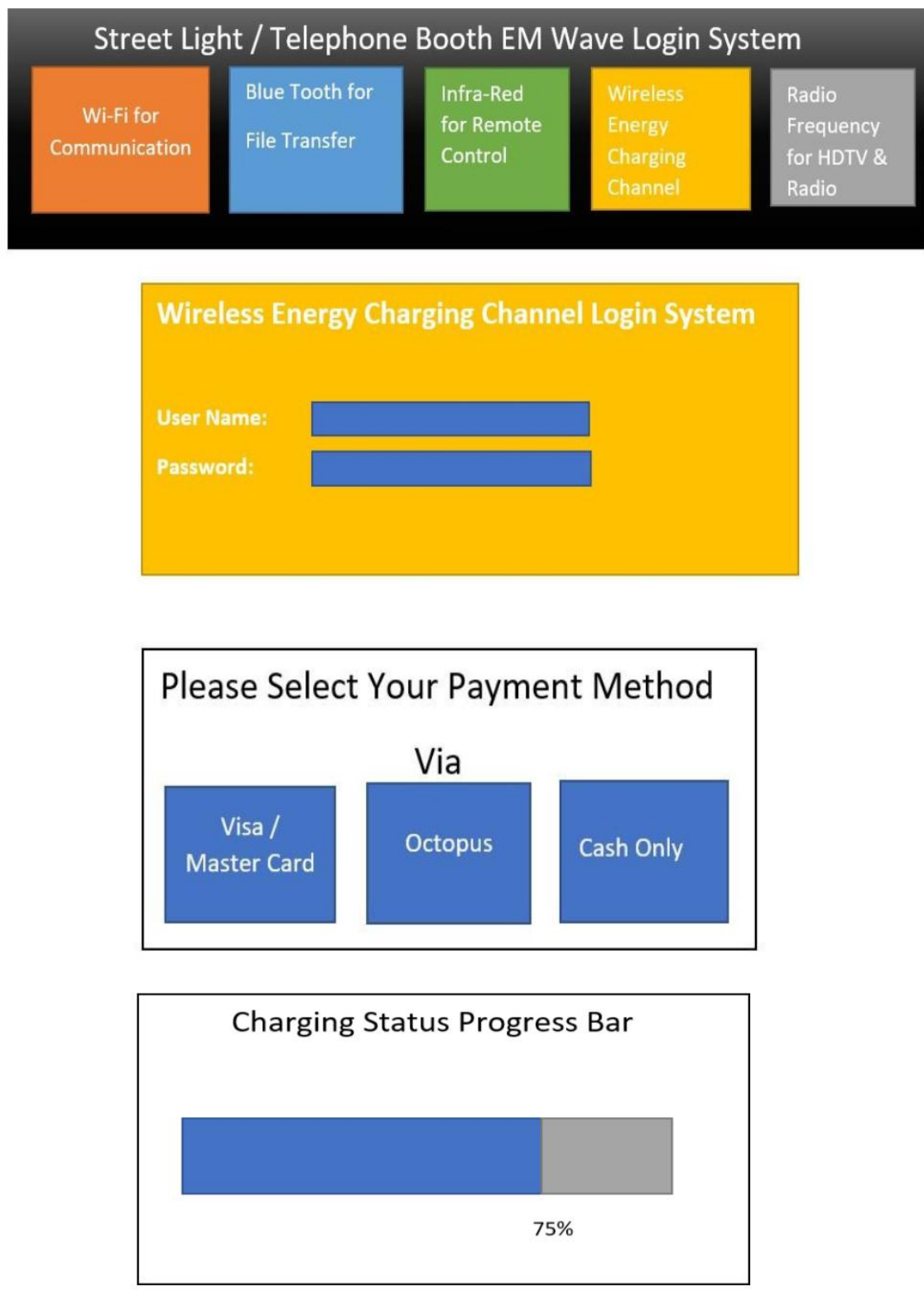

\section{Appendix Five - Antenna's Mathematical Details}

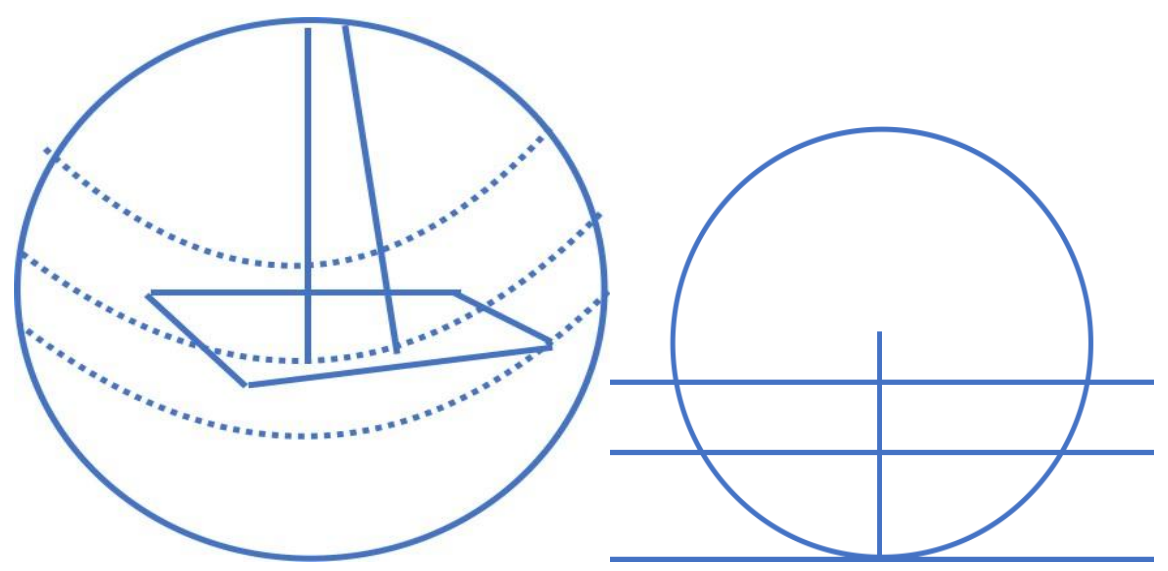

Consider a sphere with several latitudes and the one in the middle of it. If we place a square plane on it, the distance between the transmitter and the receiver antenna can be approximated. When furthermore, one consider the plane as a secant to the circle as shown on the right, one may push it such that it gradually 
approaches (like taking limiting values) to the pole of the circle. Then for the distance of the receiver sphere's pole to the transmitter's antenna, it is just the closest approximate shortest distance from the pole's square plane to the transmitter's antenna. From Ali et al., 2015 [5], it shows that directivity gain attained its highest value at the pole, thus it is natural for us to place a meta-material coated small plane near to the receiver's sphere pole so that one may maintain the shortest distance (together with highest energy harvested) from the transmitter. Indeed, according to Ali et al., 2015 [5], low incident energy power can be achieved if an array of antenna is used. In addition, when they are coated with EM meta-materials on the surface, the energy harvesting result may become much better [13].

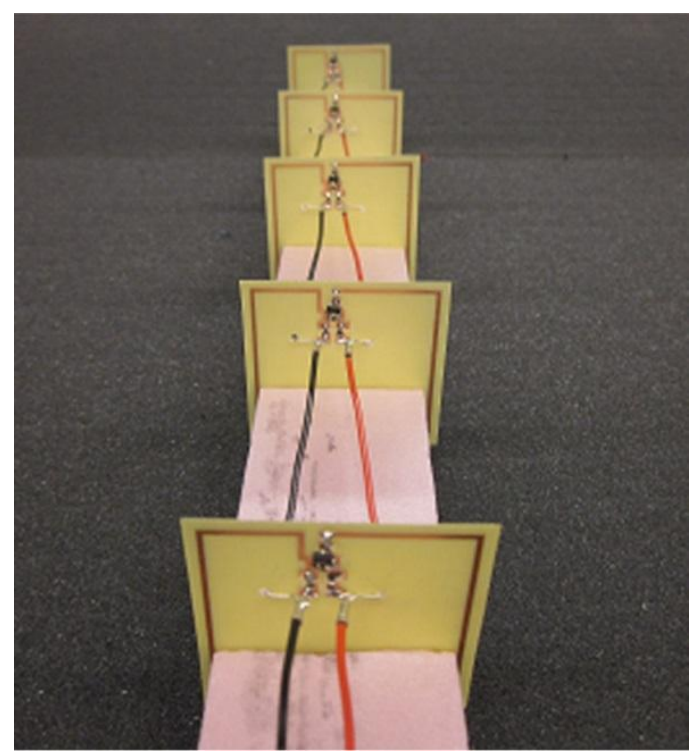

5 X 1 array of power harvesting SRRs [13].

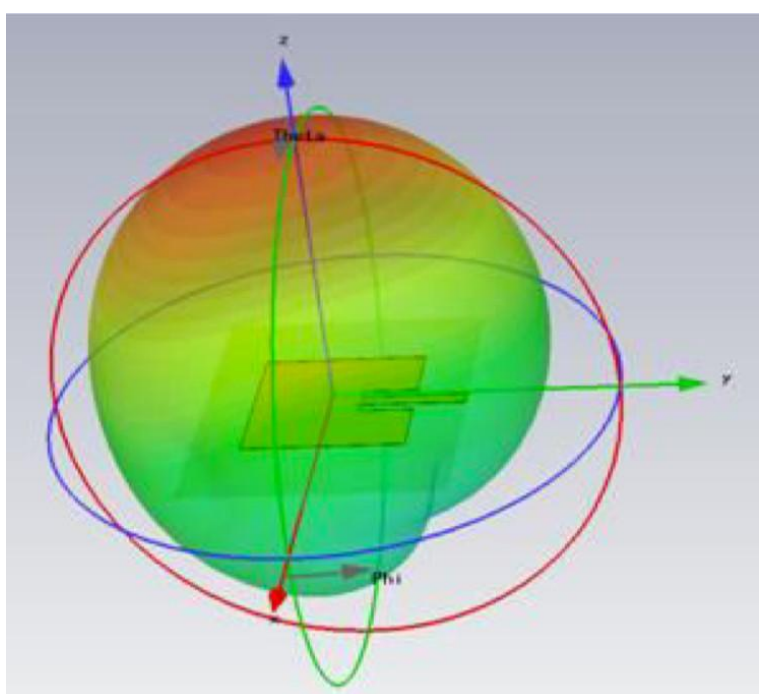

Far field of the micro strip patch antenna at frequency $0.9 \mathrm{GHz}$
15. Appendix Six - Typical RF amplifier and rectifier circuits

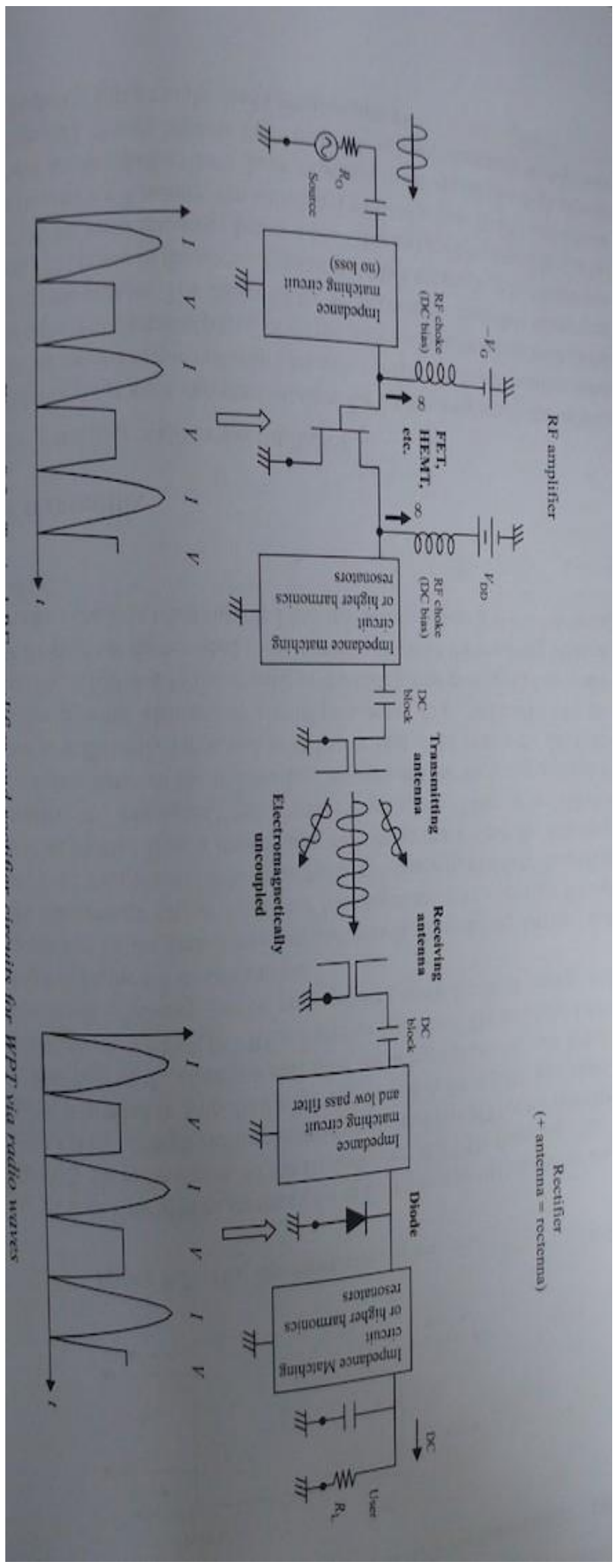




\section{Appendix Seven - Elements of the RF circuit and amplifier circuit}

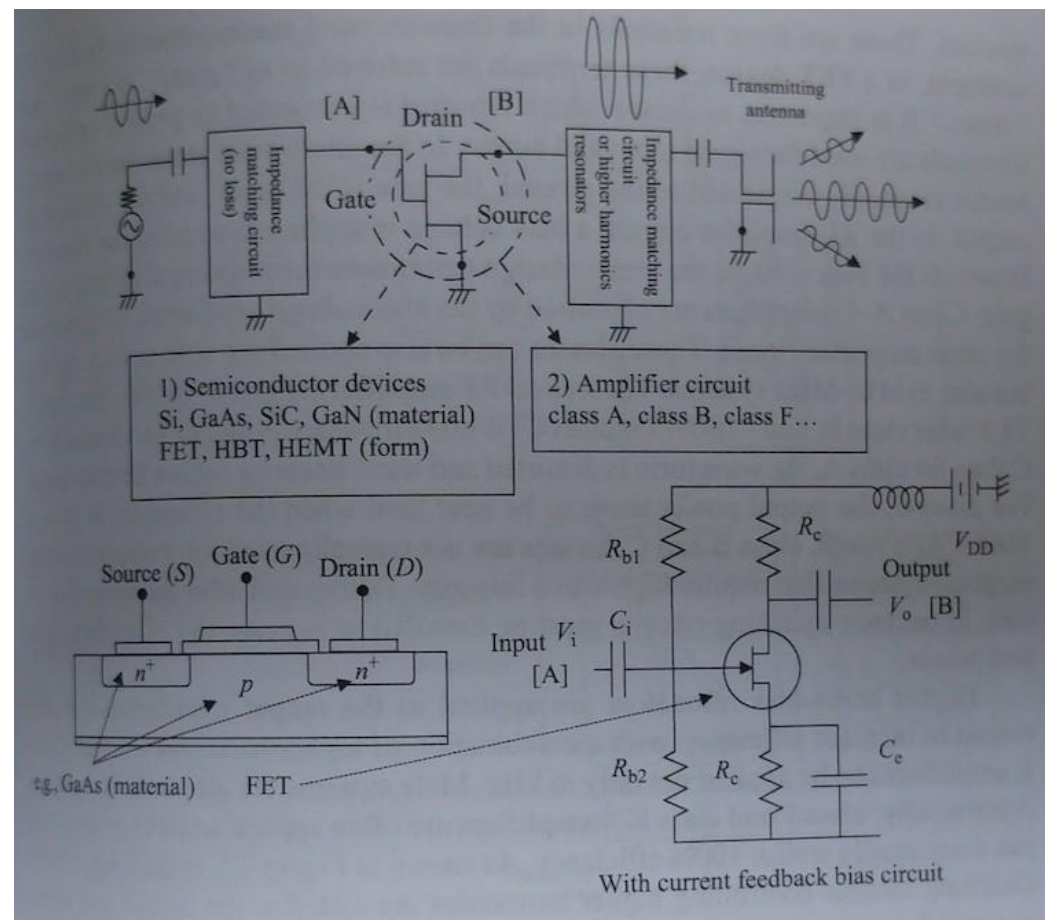

Appendix six shows a typical RF amplifier and rectifier circuits for WPT via radio waves.

Appendix seven tells elements of the RF amplifier: (1) semiconductor device and (2) amplifier circuit [14].

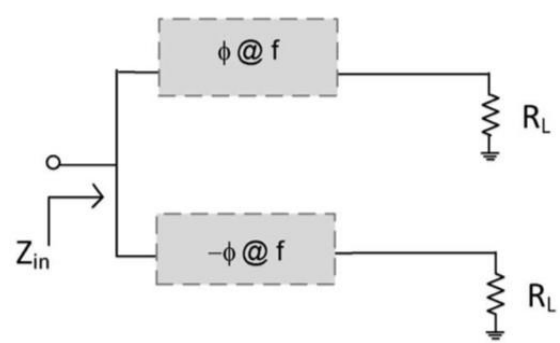

(a)

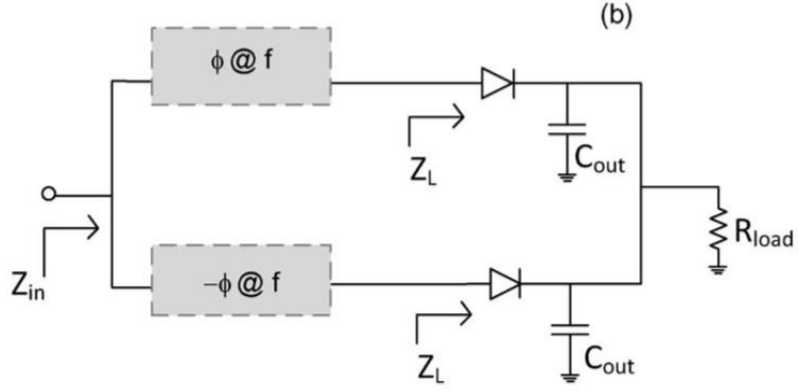

(c)

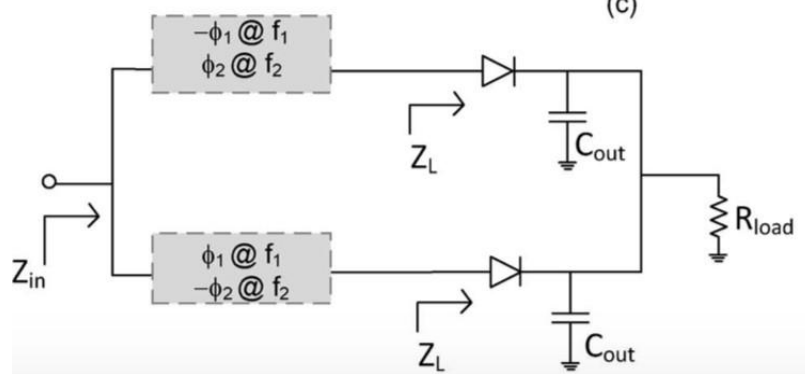

Fig-1: RCN topologies: (a) single-frequency RCN with real load; (b) single-frequency RCN-based rectifier; and (C) dualfrequency $\mathrm{RCN}$ - based rectifier [8] 


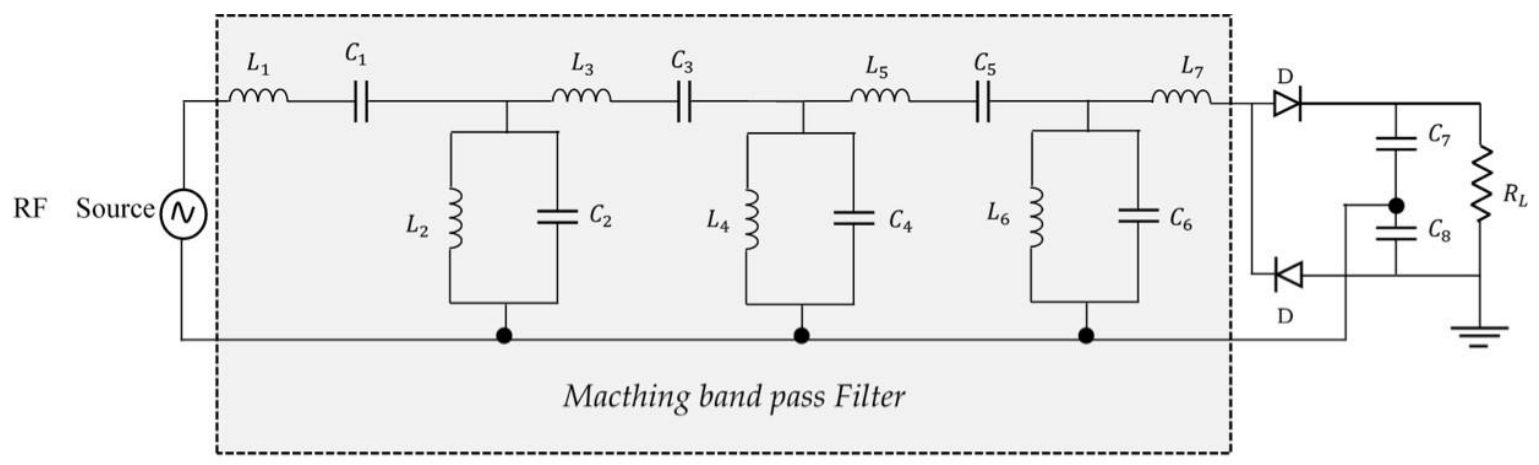

Fig-2: Rectifier with matching circuit [7]

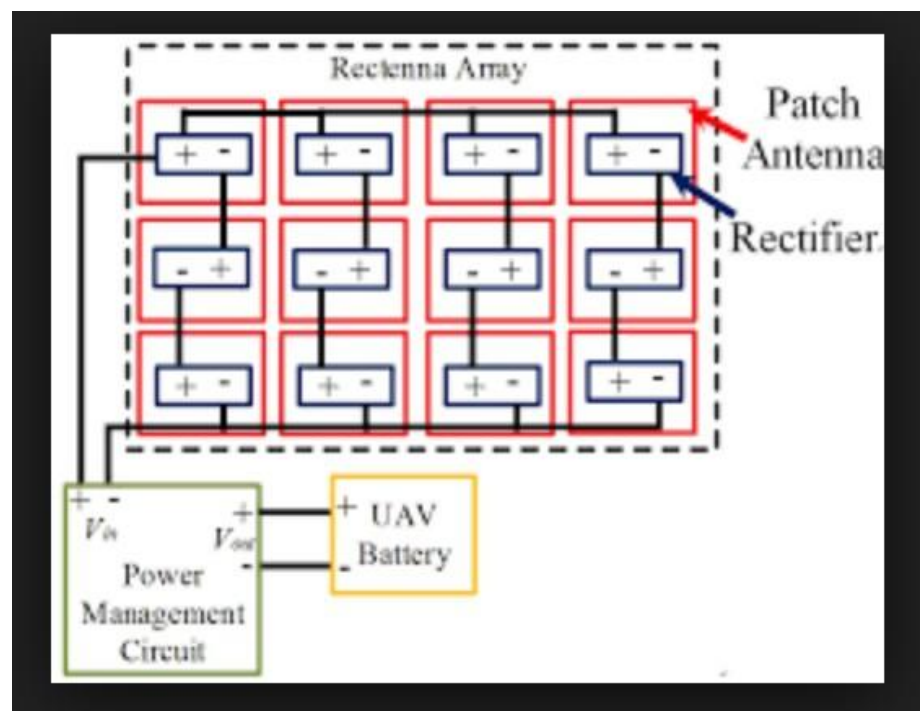

Fig-3: A set of array of patch antenna with rectifier (Rectenna array) - I suppose to add a set of matching circuit array inbetween and the energy efficiency may be maximised more than $90 \%$ [6]

\section{REMARKS}

Field Effect Transistor (FET) or Metal-OxideSemiconductor-Field Effect Transistor (MOSFET):

Suppose we use the microwave as the energy transmitter This author proposes that in this paper's thought wireless charging project, there is a possibility for one to employ the FET or the MOSET as part of the electromagnetic waves receiver [15]. The advantage is that the FET has a very high input impedance even at low frequencies (as microwave employs lower one when compared with high frequency Gramma ray etc). Hence, there will not be a voltage drop due to divider effect w.r.t. the source impedance. In order to amplify the power transferring, one should implement another transistor circuit behind the former FET receiver circuit for the amplification of the receiver's current. Then the current induced from the receiver's transmitted voltage will be amplified on the output. The output power will attain a maximum as the final output current is now being amplified (Note: $\mathrm{P}=\mathrm{VI}$ and high impedance implies high voltage and low current). Hence, one can ensure the best power outcome during the charging process for our deserved electrical devices. Indeed, the gain of my proposed circuit is:

Voltage Gain $\mathrm{G}=$ change in $\mathrm{V}_{\text {out }} /$ change in $\mathrm{V}_{\text {in }}=-\left(\mathrm{R}_{\mathrm{C}} / \mathrm{R}_{\mathrm{E}}\right)$

Gain in power for my proposed circuit is:

$$
\mathrm{P}_{\text {out }} / \mathrm{P}_{\text {in }}=\mathrm{G}^{2}
$$

It is observed that both the power gain and the voltage gain are independent of the current amplification factor. The energy gain comes from the battery (or the capacitor) located on the circuit. However, the voltage gain from a transistor is unstable [16] and thus I propose another kind of the receiver circuit with the inverting operational amplifier installed.

My thought microwave receiver circuit with the FET and the transistor will then be shown in the following (Suppose microwave is used as the source to the circuit): 


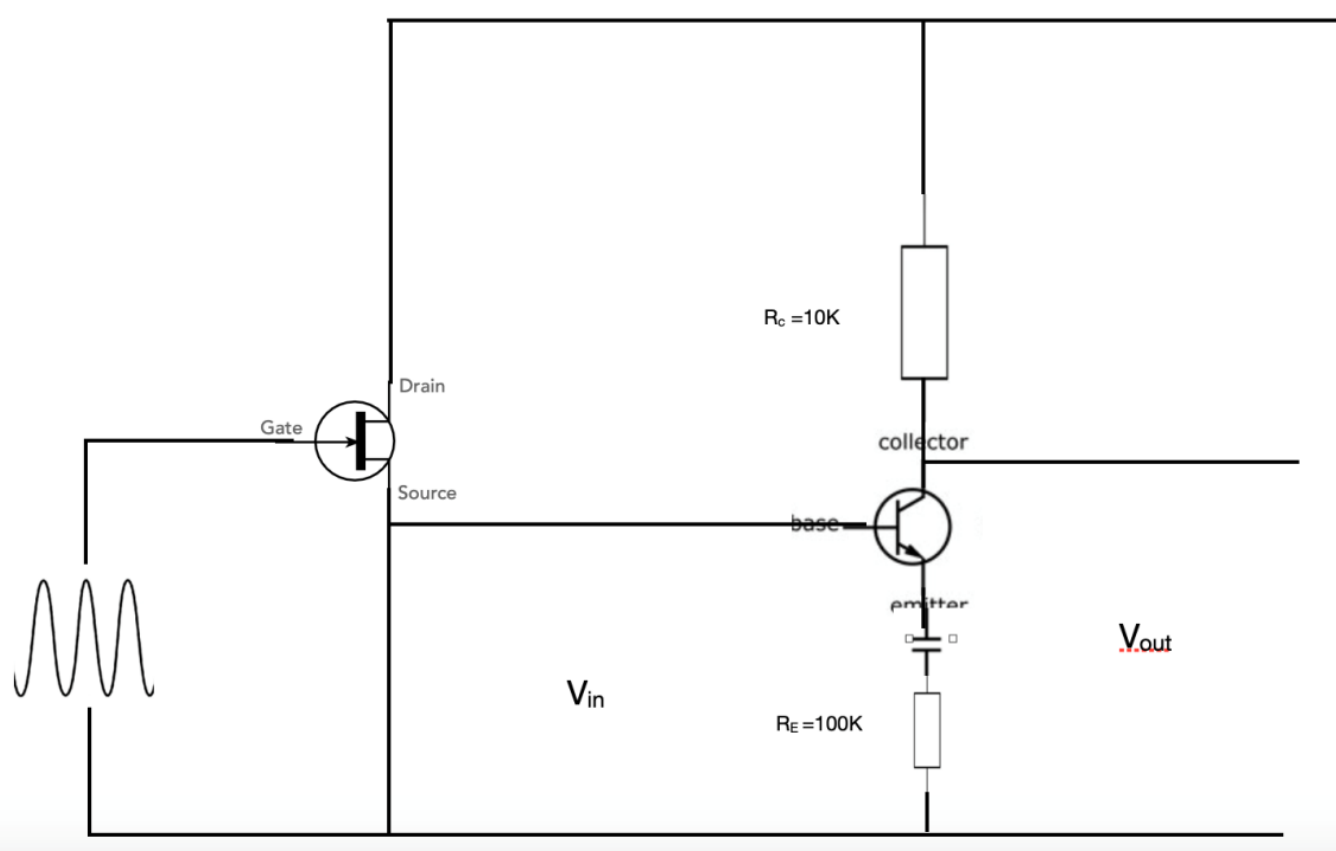

The modified receiver circuit with the inverting operational amplifier is shown below:

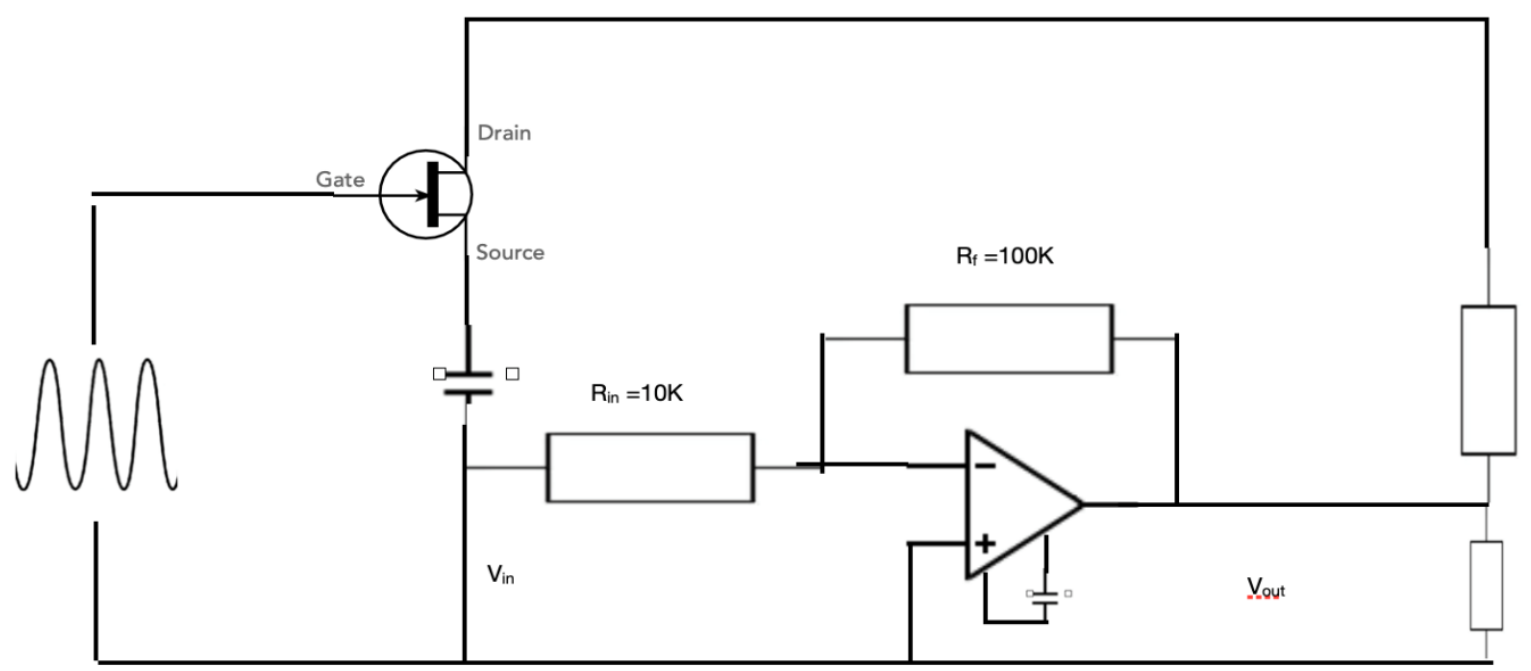

I note that the voltage gain for the modified circuit is: $\mathrm{G}$ $=\mathrm{R}_{\mathrm{f}} / \mathrm{R}_{\text {in }}$

With the feedback parameter equals to: $1 / \mathrm{G}$ or $\mathrm{R}_{\mathrm{in}} / \mathrm{R}_{\mathrm{f}}$.

In this case, one can easily control the power gain by varying the ratio of the feedback resistor to the input resistor.

2. Advance development in the materials of producing solar panel

According to the journal, nature photonics [17], scientists can use the strain engineered material like $\mathrm{MoS}_{2}$ - a kind of monolayer which can capture a broad range of solar spectrum with concentrated excitons or charged carriers to act as a photovoltaic device for this wireless charging project. This event can ensure the maximum usage of our solar energy that transmitted into our Earth and is more environmental friendly the energy loss will be less than traditional solar panels.

3. Wireless charging the Robot

Once we have the circuit technology of wireless charging, advancement in the materials of producing new solar panel together with the metamaterial coated inside the robot, we can charge the robot in anywhere and at any time. It should be note that one may convert the electrical power back into the electromagnetic wave such as the microwave. The theory is something like the microwave gun with the major component is magnetron - but suffix to the fact that it cannot be used in the cramped space for energy 
emission. Or we may find the way from our wireless router etc. But this is out of the scope of my present paper. I should note that both the power converting to electromagnetic wave and the electromagnetic wave to electrical power constitute a kind of philosophy.

\section{REFERENCES}

1. Berg, B. L., Lune, H., \& Lune, H. (2012). Qualitative Research Methods for the Social Sciences (8th ed.). Boston. 3.

2. Lam, K. S. (2018). A Quantum Look into Education, Scholars' Press.

3. Seidman, I. (1998). Interviewing as qualitative research. New York, NY: Teacher College Press.

4. Cool, C., \& Xie, H. (2000). Patterns of information use, avoidance and evaluation in a corporate engineering environment. Proceedings of American Society of information Science Annual Meeting. USA, 37, 462-472.

5. Ali, E. M., Yahaya, N. Z., Perumal, N., \& Zakariya, M. A. (2015). Design and development of harvester rectenna at GSM band for battery charging applications. ARPN Journal of Engineering and Applied Sciences, 10(21).

6. Li, K. R., See, K. Y., Koh, W. J., \& Zhang, J. W. (2017, November). Design of $2.45 \mathrm{GHz}$ microwave wireless power transfer system for battery charging applications. In 2017 Progress in Electromagnetics Research Symposium-Fall (PIERS-FALL) (pp. 2417-2423). IEEE.

7. Mouapi, A., \& Hakem, N. (2018). A new approach to design autonomous wireless sensor node based on RF energy harvesting system. Sensors, 18(1), 133.
8. Niotaki, K., Collado, A., Georgiadis, A., Kim, S., \& Tentzeris, M. M. (2014). Solar/electromagnetic energy harvesting and wireless power transmission. Proceedings of the IEEE, 102(11), 1712-1722.

9. Han, Y., Leitermann, O., Jackson, D. A., Rivas, J. M., \& Perreault, D. J. (2018). Resistance Compression Networks for Radio-Frequency Power Conversion; Institute of Electrical and Electronic Engineers http://dx.dio.org/10.1109/TPEL.2006.886601

10. Chang, A. J., Chronis, N., Karow, D. S., Marletta, M. A., \& Bargmann, C. I. (2006). A distributed chemosensory circuit for oxygen preference in $\mathrm{C}$. elegans. PLoS Biol, 4(9), e274.

11. https://www.livescience.com/51925-magneticwormhole-created.html

12. Zbitou, J., Latrach, M., \& Toutain, S. (2006). Hybrid rectenna and monolithic integrated zerobias microwave rectifier. IEEE Transactions on Microwave Theory and Techniques, 54(1), 147152.

13. Hawkes, A. M., Katko, A. R., \& Cummer, S. A. (2013). A microwave metamaterial with integrated power harvesting functionality. Applied Physics Letters, 103(16), 163901.

14. Shinohara, N. (2018). Wireless Power Transfer Theory, Technology and Applications; The Institute of Engineering and Technology.

15. https://www.researchgate.net/publication/5043514 6_Generation_and_detection_of_Terahertz_radiati on_by_Field_EffectTransistors

16. https://ieeexplore.ieee.org/document/4056510

17. https://www.researchgate.net/publication/2567658 94_Strain-engineered_artificial_atom_as_a_broadspectrum_solar_energy_funnel 